\title{
Causes of diabetic ketoacidosis in children with long-term type 1 diabetes
}

\author{
Monika Luboch-Furmańczyk', Anna Wołoszyn-Durkiewicz' , Małgorzata Myśliwiec² \\ ${ }^{1}$ Clinic of Paediatrics, Diabetology, and Endocrinology, University Clinical Centre in Gdansk, Gdansk, Poland \\ 2Department of Paediatrics, Diabetology, and Endocrinology, Medical University of Gdansk, Gdansk, Poland
}

\section{ABSTRACT}

Introduction: Diabetic ketoacidosis (DKA) is an acute complex disorder of carbohydrate, fat, and protein metabolism as well as water-electrolyte and acid-base balance due to sudden and profound insulin deficiency. It is one of the most dangerous complications of diabetes type 1 (T1D).

Aim of the study: The study aimed to analyse the causes of DKA in patients chronically ill with T1D.

Material and methods: Records from all 41 hospitalisations (34 patients: 21 females, 13 male; seven hospitalised twice) at the Clinic of Paediatrics, Diabetology, and Endocrinology of the University Clinical Centre in Gdansk in the years 2015-2017 were retrospectively analysed. The average age of the patients was 14 8/12 \pm 3 years.

Results: The average duration of diabetes was seven years. In $78 \%$ of cases patients were treated with multiple daily dose injections, others with insulin pump. In $44 \%$ of cases mild ketoacidosis with $\mathrm{pH}$ above 7.2 , in $12 \%$ moderate, in $34 \%$ severe, and in $10 \%$ ketoacidosis with pH below 7.0 was diagnosed. Average duration of fluid and intravenous insulin therapy was 30 hours. The most common reasons for ketoacidosis were: self-control errors (98\%), omission of insulin doses (78\%), and infections (37\%). Among patients treated with insulin pump $22 \%$ indicated pump failure. In the studied group $34 \%$ of patients had psychological disorders; half of them were treated by psychiatrists. In the studied group the average HbA1c level was $11.25 \pm 2.7 \%$. Previous $\mathrm{HbA1c}$ levels were $10.3 \%$, which confirms chronically inappropriate metabolic control in the analysed group. Most of the patients regularly visited a diabetes outpatient clinic, 27\% irregularly, and 5\% did not apply for control visit during the previous years.

Conclusions: Self-control errors leading to chronic hyperglycaemias, irregular visits to a diabetology outpatient clinic, and breaks in insulin therapy were the most common causes of ketoacidosis in patients with long-term type 1 diabetes. Most of the patients were teenagers a few years after diagnosis of diabetes with coincidence of psychological and psychiatric disorders.

\section{KEY WORDS:}

children, diabetes type 1, diabetic ketoacidosis, complication of diabetes.

\section{INTRODUCTION}

Diabetic ketoacidosis (DKA) is an acute complex disorder of carbohydrate, fat and protein metabolism as well as water-electrolyte and acid-base balance due to sudden and profound insulin deficiency. It is one of the most dangerous complications of diabetes type 1 (T1D).

The biochemical criteria for the diagnosis of DKA include:

\section{ADDRESS FOR CORRESPONDENCE:}

Monika Luboch-Furmańczyk, Clinic of Paediatrics, Diabetology, and Endocrinology, University Clinical

Centre in Gdansk, 7 Dębinki St., 80-952 Gdansk, Poland, e-mail: monika_anna@gumed.edu.pl 
- hyperglycaemia (blood glucose $>200 \mathrm{mg} / \mathrm{dl}$; $>11 \mathrm{mmol} / \mathrm{l})$,

- venous $\mathrm{pH}<7.3$ or bicarbonate $<15 \mathrm{mmol} / \mathrm{l}$,

- ketonemia or ketonuria $[1,2]$.

DKA may develop in patients with newly recognised diabetes as well as in patients with a previous diagnosis of the disease. The risk factors for DKA described in the literature are insulin dose omission, poor metabolic control, previous episodes of DKA, gastroenteritis with vomiting and inability to maintain hydration, psychiatric (including eating) disorders, poor social circumstances, peripubertal and adolescent period in girls, limited access to medical services, and failures in insulin pump therapy $[1,2]$.

DKA is one of the most serious complications of type 1 diabetes (T1D). Comprehensive management of DKA requires identification of the cause of DKA episode and a trial implementation of preventive treatment [1].

\section{AIM OF THE STUDY}

The study aimed to analyse the causes of DKA in patients chronically ill with T1D hospitalised at the Clinic of Paediatrics, Diabetology, and Endocrinology of the University Clinical Centre in Gdansk in the years 2015-2017.

\section{MATERIAL AND METHODS}

Researchers analysed every case of DKA needing hospitalisation in patients affected with T1D for more than a month. The study was conducted in the period from January 2015 to June 2017. The research was observational and retrospective. Microsoft Excel and Statistica were used for statistical analysis. Data were collected from medical files. Altogether, records from all 41 hospitalisations were analysed. The study group consisted of 34 patients: 21 female and 13 male. In the considered period of time seven patients were hospitalised twice due to DKA. The mean age of the patients during hospitalisation was 14 years 8 months \pm 3 years, median age 15 years 5 months (6 years 7 months -17 years 8 months). The mean time from diagnosis of T1D was 7 years \pm 3 years, median 7 years (1-14 years). Complete observation time lasted 2 years and 6 months.

\section{RESULTS}

In $78 \%$ of cases ( 32 hospitalisations) patients were treated with multiple daily dose injections before admission to the hospital, while the rest of the study group (22\%, nine hospitalisations) were treated with insulin pump therapy. At admission to the hospital, in $44 \%$ of cases (18 hospitalisations) DKA was classified as mild with $\mathrm{pH}$ over 7.2, in $12 \%$ (5) as moderate ( $\mathrm{pH} 7.1-7.2$ ), while the rest of the patients were diagnosed with severe DKA including 10\% (4) of cases with $\mathrm{pH}<7$. Rehydration and intravenous insulin therapy, implemented in order to restore fluid-electrolyte and acid-base balance, lasted on average 30 hours. One patient needed 81 hours of intensive intravenous treatment. Four patients were hospitalised in an intensive care unit during the first days of treatment. Mistakes in self-control, reported by $98 \%$ of patients ( 33 children), were the most frequent cause of DKA in the study group. (Tables 1 and 2). In one case (eight-year-old girl affected with diabetes since the age of five years) the parents maintained that they had strictly followed the recommendations given by a diabetologist. The patient was hospitalised due to an acute infection complicated with mild DKA. In 78\% of cases (32 hospitalisations), patients omitted a dose of insulin before hospitalisation, did not administer long-acting insulin, or ate a meal without blood glucose level measurement and insulin administration.

Only in $37 \%$ of cases (15 hospitalisations) an acute infection was diagnosed at the admission to the hospital, which could have led to metabolic decompensation. Caregivers of patients suffering from T1D are educated on insulin dose adaptation during acute infections; however, in the presented cases they did not follow the recommendations properly. In three cases, adolescents, diagnosed with an infection, were not under the care of their parents or their parents were not aware of their health status. Patients, on the other hand, did not take the appropriate measures, such as additional insulin dose administration, additional blood glucose measurements, diet modification, or basal insulin adjustment in an insulin pump, in order to prevent DKA development. An infection changes insulin requirements and insulin sensitivity. Despite infection, patients did not consult a doctor early enough.

In the group of patients treated with continuous subcutaneous insulin infusion (CSII) only in two cases (5\% of all hospitalisations) did researchers report insulin pump failure or occlusion of an infusion set resulting in DKA.

In the study group the mean glycated haemoglobin level (HbAlc) during hospitalisation was $11.25 \pm 2.7 \%$, whereas the mean $\mathrm{HbA} 1 \mathrm{c}$ level obtained from the previous measurement, carried out in an outpatient clinic

TABLE 1. The prevalence of risk factors for diabetic ketoacidosis in the study group

\begin{tabular}{|l|c|c|}
\hline Risk factor & $\begin{array}{c}\text { Number } \\
\text { of cases }(\mathrm{N}=41)\end{array}$ & $\begin{array}{c}\text { Percentage } \\
\text { of cases }\end{array}$ \\
\hline Mistakes in self-control & 40 & 98 \\
\hline $\begin{array}{l}\text { Psychological or psychiatric } \\
\text { disorders or drug/alcohol abuse }\end{array}$ & 16 & 39 \\
\hline Acute infection & 15 & 37 \\
\hline $\begin{array}{l}\text { Irregular appointments in the } \\
\text { outpatient clinic }\end{array}$ & 13 & 32 \\
\hline Lack of parenting control & 14 & 34 \\
\hline $\begin{array}{l}\text { Insulin pump failure } \\
\text { or occlusion of infusion set }\end{array}$ & 2 & 5 \\
\hline
\end{tabular}


TABLE 2. Diagnostic criteria for diabetic ketoacidosis risk factors adopted in the study

\begin{tabular}{|c|c|}
\hline Risk factor & Diagnostic criteria (at least one of listed) \\
\hline Mistakes in self-control & $\begin{array}{l}\text { - less than } 4 \text { blood glucose measurements per day } \\
\text { - lack of insulin adjustment in case of increased requirements (e.g. due to the fever) } \\
\text { - unspecified amount of consumed carbohydrates } \\
\text { - meal consumption without required insulin administration } \\
\text { - omission of basal insulin pump } \\
\text { - handling incompatible with manufacturer's instructions (e.g. irregular exchange of infusion sets) }\end{array}$ \\
\hline $\begin{array}{l}\text { Psychological or psychiatric } \\
\text { disorders or drug/alcohol abuse }\end{array}$ & $\begin{array}{l}\text { - every psychological or psychiatric disorder confirmed by psychiatrist or psychologist } \\
\text { - use of psychoactive substances or alcohol abuse }\end{array}$ \\
\hline Acute infection & - acute infection with or without fever \\
\hline $\begin{array}{l}\text { Irregular medical appointment } \\
\text { in the outpatient clinic }\end{array}$ & - less than 4 medical appointments per year \\
\hline Lack of parenting control & $\begin{array}{l}\text { - lack of parental supervision of blood glucose measurements/glycaemic values and insulin admini- } \\
\text { stration (also lack of glucometer/self-control diary/insulin pump records supervision by parents/ } \\
\text { caregivers) } \\
\text { - long-term absence of trained caregiver (e.g. during summer camp, holidays with extended family } \\
\text { members, sleepover at friend's house) } \\
\text { - run away from home }\end{array}$ \\
\hline
\end{tabular}

or during the last hospitalisation, was $10.3 \%$. This fact highlights the poor metabolic control in the study group. HbAlc was $7.3 \%$ only in two patients (6\%), who developed mild DKA due to an insulin pump failure or an occlusion of insulin set, while the rest of the patients presented higher levels of HbAlc.

A substantial proportion of patients attended medical appointments in the outpatient clinic regularly, at least four times a year, in accordance with the guidelines [3], $27 \%$ (11 patients) irregularly, while $5 \%$ (2 patients) of them had not seen a diabetologist in the past few years prior to hospitalisation.

In $34 \%$ of cases ( 14 hospitalisations) psychological or psychiatric disorders were reported, most frequently de-

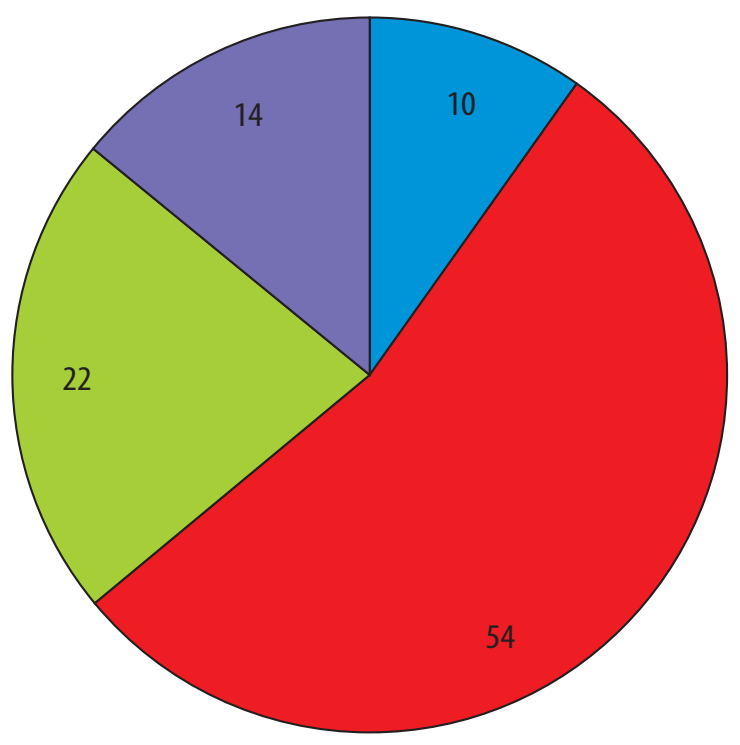

[\%]

$\square 1 \quad \square 2 \quad \square 3 \quad \square 4$

FIGURE 1. Coexistence of risk factors: number of identified risk factors in patients with diabetic ketoacidosis pression, addictions, or other behaviour disorders, and half of this group were treated psychiatrically. Researchers observed the lack of parenting control in 35\% (12) of patients, mostly teenagers, which led to numerous problems in self-control of diabetes. Moreover, conflicts between a child and parents were frequent, resulting in difficulties in self-control.

As mentioned above, in the considered period of time seven (20\%) patients (four girls and three boys) were hospitalised twice due to DKA. Serious failures of self-control caused by psychoactive substances and alcohol intake or resulting from the lack of parental care were the major problems in this group of patients. These patients did not perform blood glucose measurements chronically, and in the days preceding the hospitalisation they did not administer insulin.

One patient, a 13-year-old boy, suffering from diabetes since the age of one year, was from a dysfunctional family. The patient presented significant behavioural disorders related to drug addiction and had been in trouble with the law previously. His $\mathrm{HbA1c}$ was $17.3 \%$ and this result was the highest in the whole study group. After being hospitalised for DKA twice, a week apart, the patient was placed in an educational institution under court order and with full acceptance of his mother. According to data received from follow-up observations, the patient's glycaemic and metabolic control was significantly improved.

In the vast majority of cases, risk factors for DKA overlapped with one another. Negligence and self-control failures were associated most frequently with psychological disorders, lack of parental control, acute infections, and irregular diabetes visits. Researchers identified two risk factors for DKA in 22 cases (54\%), three factors in nine cases $(22 \%)$, and in six cases (14\%) four risk factors were found (Fig. 1). Only in four patients (10\%) was there an isolated risk factor for DKA, in three cases mistakes 
in self-control were reported, and in one case an acute infection was diagnosed.

\section{DISCUSSION}

Annually, in the Clinic of Paediatrics, Diabetology, and Endocrinology of the University Clinical Centre in Gdansk, 130 patients are hospitalised due to newly recognised diabetes. The vast majority of this group receive professional care at the Outpatient Diabetology Clinic for children. Follow-up appointments are arranged every 1-3 months, depending on the patients' requirements. In addition, patients have the possibility to consult a psychologist and a dietician. According to data analysis, these actions allow acute complications of diabetes to be avoided, including DKA. Unfortunately, despite meticulous specialist care, several patients are admitted to the hospital with a diagnosis of DKA every year. On account of the risk of serious complications of DKA, including cerebral oedema and death [4], it is crucial to look for possible causes of this state and implement preventive measures if necessary.

According to literature data, adolescents, primarily female, suffering from diabetes for several years are most likely to develop DKA, apart from patients with newly recognised diabetes [5-7]. Researchers obtained similar results in the presented study. According to the research by Cho et al., poor metabolic control in adolescence constitutes a risk factor for late diabetic complications. Moreover, in the aforementioned studies, researchers emphasised the fact that puberty carries a greater risk for nephropathy and vasculopathy in diabetic patients compared to patients who develope diabetes after puberty [6]. For this reason, meticulous self-control and consequently good metabolic control have a pivotal role in this group of patients. According to the guidelines, the target $\mathrm{HbAlc}$ level in patients with T1D is $\leq 6.5 \%$ unless associated with episodes of hypoglycaemia or compromised quality of life. In other cases, the therapeutic goal should be $\leq 7 \%$ [3]. Unfortunately, in the study group, the mean $\mathrm{HbAlc}$ level was well above normal range. Moreover, the same observation was also noticed several months before developing a DKA episode. It demonstrates poor metabolic control in the study group and a hyperglycaemic tendency sustaining for many months or even years. Elevated HbA1c level, most frequently observed in adolescents with type 1 diabetes, correlates with higher risk of developing DKA $[5,7,8]$. Lack of proper glycaemic control does not protect patients from severe hypoglycaemic episodes [7]. Furthermore, considerable daily glycaemic fluctuations increase the risk of late-onset complications of diabetes such as nephropathy, retinopathy, neuropathy, and cardiovascular disorders. For comparison, in another multicentre study, carried out in several Clinical Centres in Poland, including the one in Gdańsk, the mean HbAlc level in children chronically affected with T1D was
$7.6 \pm 1.5 \%$. In the study group the mean age of patients was $13.04 \pm 3.43$ years at that time, and the mean disease duration was $6.93 \pm 2.33$ years [9].

Mistakes in self-control were the most frequent direct cause of DKA in the study group. Patients and their caregivers admitted omitting insulin doses comprising long-acting insulin (basal insulin). Other mistakes included lack of blood glucose measurements and improper insulin dosage adjustment. A large group of patients administered insulin without calculation of carbohydrate units or consumed meals without insulin administration, intentionally or not. Subsequently, these actions led to hyperglycaemia and DKA development.

Parents of diabetic patients undergo educational training on self-management in diabetes. The aim of the education is to prevent paediatric patients from making the most common mistakes. However, frequently children are too young to fully participate in the training. Consequently, later, in adolescence, they do not adhere to medical recommendations given by their parents or doctors, and they deceive caregivers. Despite a willingness to take responsibility for their thwerapy and eagerness to participate in re-education programs in an outpatient clinic or at the Department of Diabetology, they are often not mature enough or psychologically ready.

During the difficult period of puberty, when adolescents seek their place in society, diabetes, which is a chronic disease, becomes a serious problem. Patients try to forget about their medical condition, and as a result, they start taking drugs, ignore glycaemic control, or omit insulin doses. Furthermore, parent-adolescent conflict, frequently observed in this period of life, may interfere with proper self-management in diabetes $[10,11]$.

Psychological care, systematic re-education, and motivating patients to improve self-control are particularly relevant in this stage of disease in order to prevent DKA episodes. Meetings of patients and their families in support groups may be helpful. It is significantly important in light of data showing that $34 \%$ of patients suffered from psychological or psychiatric disorders like depression, addictions, and behaviour disorders. Half of the group was treated psychiatrically. In 2007, Delatamer, in a study entitled "Psychological care of children and adolescents with diabetes", paid attention to the fact that there is a greater incidence of psychiatric disorders in patients affected with type 1 diabetes [12]. Female patients, patients with poor metabolic control and recurrent DKA episodes are more predisposed to develop psychiatric disorders. Eating disorders and depression were observed most frequently and were associated with poor glycaemic control and an increased number of hospitalisations $[12,13]$. According to the reports from 2016, the prevalence of severe psychosocial illness in patients with T1D was $20.24 \%$. The most frequent psychosocial malfunction was irritation (38.1\%), followed by depression $(36.9 \%)$, and anxiety (32.1\%) [14]. 
Social conditions [8] and family situation of the patient, including the quality of care provided by parents or legal guardians, are among the most important factors affecting metabolic control in diabetes and the incidence of DKA episodes [15]. In the study group 34\% of DKA episodes were observed in periods without direct parental supervision, for example during holiday trips or sleepover at a friend's house. There were also cases of parental neglect including alcoholic families and families incapable of raising a child. Several patients, after a severe episode of DKA, were placed in educational institutions or in foster families by the family court. According to received data, insulin pump failure or insulin set occlusions rarely lead to DKA development. Therefore, it helps to reduce unnecessary fears regarding this model of therapy. The vast majority of patients were treated with simple insulin injections ("pen therapy"), and DKA episodes were not related to equipment failure. Similar data was obtained in studies carried out in Austria and Germany, in which researchers did not observe greater incidence of DKA in patients treated with CSII, despite the risk of insulin pump failure or infusion set occlusions [7]. Moreover, according to literature data, paediatric patients treated with CSII therapy present lower levels of HbA1c in comparison with patients treated with multiple daily dose injections [8]. In addition, in patients on insulin pump therapy using continuous glucose monitoring (CGM), researchers reported not only improvement in glycaemic control but also a higher quality of life [16]. Better quality of life is significantly important for children with chronic diseases like diabetes.

\section{CONCLUSIONS}

The most frequent causes of DKA in patients with T1D, lasting for more than a month, are mistakes in self-control leading to chronic metabolic decompensation, discontinuous administration of insulin resulting indirectly in DKA development, and missing appointments with a diabetologist. The majority of patients are adolescents after several years of disease duration, frequently affected concomitantly with psychiatric and psychological problems. Further analysis of DKA causes and implementation of preventive measures are required.

On the basis of literature data and experience obtained by researchers from the Clinical Centre in Gdańsk, the authors suggest the implementation of preventive measures concerning education and re-education programmes in the field of nutrition and physical activity, as well as self-management during infections and fast identification of concomitant diseases in patients with diabetes. Constant dietary and psychological care, together with support group formation, appear to be necessary. It is imperative to react immediately to any potential symptoms of psychological disorders to which patients with T1D are predisposed. It is vital to build a continuous partnership between paediatricians or general practitioners and specialists in the area of management with children affected by diabetes.

\section{DISCLOSURE}

The authors declare no conflict of interest.

\section{REFERENCES}

1. Wolfsdorf JI, Allgrove J, Craig ME, et al. A Consensus Statement from the International Society for Pediatric and Adolescent Diabetes: Diabetic ketoacidosis and hyperglycemic hyperosmolar state. Pediatr Diabetes 2014; 15 (Suppl 20): 154-179.

2. Alois CI, Rizzolo D. Diabetic ketoacidosis: Heralding type 1 diabetes in children. JAAPA 2017; 30: 20-23.

3. 2017 Guidelines on the management of diabetic patients. A position of Diabetes Poland. Clin Diabet 2017; 6 (Suppl A): A1-A80.

4. Wasag DR, Gregory JW, Dayan C, et al. Excess all-cause mortality before age 30 in childhood onset type 1 diabetes: data from the Brecon Group Cohort in Wales. Arch Dis Child 2018; 103: 44-48.

5. Rica I, Mingorance A, Gómez-Gila AL, et al. Achievement of metabolic control among children and adolescents with type 1 diabetes in Spain. Acta Diabetol 2017; 54: 677-683.

6. Cho YH, Craig ME, Donaghue KC. Puberty as an accelerator for diabetes complications. Pediatr Diabetes 2014; 15: 18-26.

7. Cengiz E, Xing D, Wong JC, et al. Severe Hypoglycemia and Diabetic Ketoacidosis among Youth with Type 1 Diabetes in the T1D Exchange Clinic Registry. Pediatr Diabetes 2013; 14: 447-454.

8. Zuijdwijk CS, Cuerden M, Mahmud FH. Social Determinants of Health on Glycemic Control in Pediatric Type 1 Diabetes. J Pediatr 2013; 162: 730-735.

9. Jarosz-Chobot P, Polanska J, Myśliwiec M, et al. Multicenter cross-sectional analysis of values of glycated haemoglobin (HbAlc) in Polish children and adolescents with long-term type 1 diabetes in Poland: PolPeDiab study group. Pediatr Endocrinol Diabetes Metab 2012; 18: 125-129.

10. Dashiff C, Hardeman T, McLain R. Parent-adolescent communication and diabetes: an integrative review. J Adv Nurs 2008; 62: 140-162.

11. Luyckx K, Seiffge-Krenke I, Missotten L, et al. Parent-adolescent conflict, treatment adherence and glycemic control in Type 1 diabetes: The importance of adolescent externalising symptoms. Psychol Health 2013; 28: 1082-1097.

12. Delamater AM. Psychological care of children and adolescents with diabetes. Pediatr Diabetes 2007; 8: 340-348.

13. Alvar CM, Coddington JA, Foli KJ, et al. Depression in the SchoolAged Child With Type 1 Diabetes: Implications for Pediatric Primary Care Providers. J Pediatr Health Care 2018; 32: 43-52.

14. Khandelwal S, Senger G, Sharma M, et al. Psychosocial Illness in Children with Type 1 Diabetes Mellitus: Prevalence, Pattern and Risk Factors. J Clin Diagn Res 2016; 10: 5-8.

15. Serkel-Schrama IJ, de Vries J, Nieuwesteeg AM, et al. The Association of Mindful Parenting with Glycemic Control and Quality of Life in Adolescents with Type 1 Diabetes: Results from Diabetes MILES - The Netherlands. Mindfulness 2016; 7: 1227-1237.

16. Hirose M, Beverly EA, Weinger K. Quality of Life and Technology: Impact on Children and Families with Diabetes. Curr Diab Rep 2012; 12: 711-720. 